\title{
Heart-Kidney Biomarkers in Patients Undergoing Cardiac Stress Testing
}

\section{Mikko Haapio, ${ }^{1,2}$ Andrew A. House, ${ }^{2,3}$ Massimo de Cal, ${ }^{2}$ Dinna N. Cruz, ${ }^{2}$ Paolo Lentini, ${ }^{2}$ Davide Giavarina, ${ }^{4}$ Antonio Fortunato, ${ }^{4}$ Luigi Menghetti, ${ }^{5}$ Matteo Salgarello, ${ }^{6}$ Andrea Lupi, ${ }^{6}$ Giuliano Soffiati, ${ }^{4}$ Alessandro Fontanelli, ${ }^{5}$ Pierluigi Zanco, ${ }^{6}$ and Claudio Ronco ${ }^{2}$}

\author{
${ }^{1}$ Division of Nephrology, Meilahti Hospital, HUCH, P.O. Box 340, 00029 Helsinki, Finland \\ ${ }^{2}$ Department of Nephrology, St. Bortolo Hospital, 36100 Vicenza, Italy \\ ${ }^{3}$ Division of Nephrology, LHSC, London, ON, Canada N6A 5A5 \\ ${ }^{4}$ Clinical Chemistry and Haematology Laboratory, St. Bortolo Hospital, 36100 Vicenza, Italy \\ ${ }^{5}$ Department of Cardiology, St. Bortolo Hospital, 36100 Vicenza, Italy \\ ${ }^{6}$ Department of Nuclear Medicine, St. Bortolo Hospital, 36100 Vicenza, Italy
}

Correspondence should be addressed to Mikko Haapio, mikko.haapio@helsinki.fi

Received 15 August 2010; Accepted 24 September 2010

Academic Editor: Mitchell H. Rosner

Copyright (C) 2011 Mikko Haapio et al. This is an open access article distributed under the Creative Commons Attribution License, which permits unrestricted use, distribution, and reproduction in any medium, provided the original work is properly cited.

\begin{abstract}
We examined association of inducible myocardial perfusion defects with cardiorenal biomarkers, and of diminished left ventricular ejection fraction (LVEF) with kidney injury marker plasma neutrophil gelatinase-associated lipocalin (NGAL). Patients undergoing nuclear myocardial perfusion stress imaging were divided into 2 groups. Biomarkers were analyzed pre- and poststress testing. Compared to the patients in the low ischemia group $(n=16)$, the patients in the high ischemia group $(n=18)$ demonstrated a significantly greater rise in cardiac biomarkers plasma BNP, NT-proBNP and cTnI. Subjects were also categorized based on pre- or poststress test detectable plasma NGAL. With stress, the group with no detectable NGAL had a segmental defect score 4.2 compared to $8.2(P=.06)$ in the detectable NGAL group, and 0.9 vs. $3.8(P=.03)$ at rest. BNP rose with stress to a greater degree in patients with detectable NGAL $(10.2 \mathrm{vs} .3 .5 \mathrm{pg} / \mathrm{mL}, P=.03)$. LVEF at rest and with stress was significantly lower in the detectable NGAL group; 55.8 versus $65.0(P=.03)$ and 55.1 vs. $63.8(P=.04)$, respectively. Myocardial perfusion defects associate with biomarkers of cardiac stress, and detectable plasma NGAL with significantly lower LVEF, suggesting a specific heart-kidney link.
\end{abstract}

\section{Introduction}

Bidirectional signaling between the heart and the kidneys is being increasingly recognized as an important determinant of progression of disease states in both organs. This interorgan relationship, called the cardiorenal syndromes, has been defined with a novel classification [1] and described in detail at a recent consensus conference [2]. The accurate characterization of the types of signaling and their consequences is paramount to the understanding of cardiorenal and renocardiac syndromes [1].

Both in coronary artery disease $(\mathrm{CAD})$ and congestive heart failure $(\mathrm{CHF})$ the levels of plasma B-type natriuretic peptides (BNPs) have been shown to be elevated compared to healthy controls $[3,4]$. Furthermore, BNPs have established their role in diagnosing acute heart failure $[5,6]$ and have been shown to be strong prognostic markers for mortality in CAD $[7,8]$ and CHF patients [9], with and without renal insufficiency [10-13].

Neutrophil gelatinase-associated lipocalin (NGAL) is a novel biomarker reflecting damage to renal tubular cells, with elevated levels in urine and plasma from two hours onwards after acute insult to the kidneys [14]. Moreover, the rise of NGAL is detectable substantially faster than the possible rise in plasma creatinine thus allowing diagnosis of acute kidney injury earlier, and with better sensitivity [15]. NGAL 
has been shown to be elevated also in patients with CHF, suggesting an association between cardiac and renal damage $[16,17]$.

During cardiac stress testing, a number of studies have indicated that BNPs may be higher at baseline or increase to a greater extent in those whose tests indicated the presence of ischemia $[18,19]$. No published data exist on whether inducible myocardial ischemia during cardiac stress testing can cause injurious signaling to the kidneys measured by increase in plasma NGAL.

We designed this study in patients undergoing nuclear myocardial perfusion stress imaging to examine the relationship between baseline and poststress changes in the number of myocardial perfusion defects (as a surrogate for cardiac ischemia), with levels of several cardiac biomarkers (BNP, N-terminal-proBNP and cardiac troponin I), markers of oxidative stress (advanced oxidation protein products), pre- and poststress cardiac chamber size and left ventricular ejection fraction (LVEF). We also examined for an association between cardiac ischemia and diminished LVEF, and kidney injury through measurement of the biomarker plasma NGAL.

We hypothesized that subjects demonstrating higher levels of perfusion defects would have increased poststress levels of cardiac biomarkers, altered cardiac chamber dimensions, lower LVEF and increased levels of plasma NGAL.

\section{Patients and Methods}

2.1. Patients and Study Protocol. The study was performed and the blood samples collected and analyzed in San Bortolo Hospital, Vicenza, Italy, between December 2007 and February 2008. Consecutive adult patients undergoing elective nuclear stress perfusion imaging fulfilling inclusion criteria were invited to participate. Any outpatient planned to go to perfusion test was eligible for participation, regardless of the indication for the test, with the exclusion of patients with prior heart or kidney transplant. A convenience sample of 34 patients consented to the study. Nuclear stress test was performed according to hospital's normal procedure, and informed consent was obtained from all patients who participated in the study. The study was approved by the Institutional Review Board.

Subjects underwent perfusion stress imaging performed on a Tuesday of the week of enrollment, and blood samples were drawn approximately 10 minutes before, and two hours after the stress test, prior to discharge from the Nuclear Medicine department. A subsequent imaging study at rest was performed on the Friday of the same week to provide baseline perfusion images, during which no additional blood was drawn. The stress test was performed using bicycle ergometer exercise (16 patients), or pharmacologically using dipyridamole $(0.56 \mathrm{mg} / \mathrm{kg}$ intravenously in 4 minutes $)$ followed by aminophylline $120 \mathrm{mg}$ intravenously $(240 \mathrm{mg}$ if any symptoms of side effects of dipyridamole were present). The scintigraphy was performed according to EANM/ESC guidelines [20], using $99 \mathrm{mTc}$-sestamibi as tracer and a twoday gated SPECT protocol. The perfusion scan results were interpreted by two experts as per standardized guidelines for reporting [21, 22], blinded to the experimental laboratory results.

The recorded variables included date of birth, sex, age, type of perfusion study and stress (i.e., exercise or pharmacologic), cardiac risk factors, and previous known myocardial infarction or coronary artery disease. Medications that may influence BNP were recorded (nitrates, calcium antagonists, and beta blockers). The adequacy of the stress test was assessed by peak heart rate and blood pressure. Segmental myocardial perfusion defects at rest and with stress were reported per standardized guidelines as well as cardiac left ventricular end diastolic volume, end systolic volume, and ejection fraction. Changes with stress were calculated for all relevant variables. Ejection fraction and related measurements were compared between the rest and stress study results to calculate the changes (deltas).

Plasma creatinine was measured at baseline to determine the presence or absence of kidney dysfunction. Blood samples were taken pre- and postperfusion testing and analyzed for blood hemoglobin, plasma albumin, creatinine, BNP, Nterminal-proBNP (NT-proBNP), cardiac troponin I (cTnI), NGAL, and advanced oxidation protein products (AOPP). Changes in hematocrit and albumin with stress indicated acute shifts in plasma volume as previously described in [23], hence poststress values for all relevant analytes were adjusted based on delta albumin.

2.2. Specific Laboratory Techniques. Plasma BNP and cTnI were analyzed using immunochemiluminescence immunoassay and ADVIA Centaur analyzer (Siemens Healthcare Diagnostics Inc., Deerfield, IL) and plasma NT-proBNP with electrochemiluminescence immunoassay and Elecsys 2010 analyzer (Roche Diagnostics AG, Rotkreuz, Switzerland). Plasma samples for NGAL were quickly stored in minus 80 degrees Celcius for later analysis. Plasma NGAL was measured with fluorescence-based immunoassay with the Triage point-of-care analyzer (Biosite Inc., San Diego, CA, USA), which is a rapid quantitative measurement of NGAL concentration in EDTA-anticoagulated whole blood or plasma (single-use plastic cartridges with immobilized NGAL antigen and containing NGAL-specific monoclonal antibodies conjugated to fluorescent nanoparticles). The detection limits for this NGAL analysis are $60-1300 \mathrm{ng} / \mathrm{mL}$. AOPP were measured by spectrophotometry and concentrations were expressed as micromoles per liter of chloramine- $\mathrm{T}$ equivalents.

2.3. Statistical Methods. According to the median value for inducible perfusion defects, which defined two groups (low and high inducible ischemia), study parameters were compared between-groups using unpaired $t$-tests (MannWhitney $U$ test for skewed variables) or Fisher's exact test for categorical variables. Within-group changes (pre- and poststress) were analyzed using paired $t$-tests (or Wilcoxon signed rank test). Many NGAL levels were below the level of detection, and accurate pre- and postdeltas could not be calculated, hence comparisons could not be made between the low and high inducible ischemia groups. Therefore, subjects were categorized based on any level of detectable 
NGAL ( $\geq 60 \mathrm{ng} / \mathrm{mL}$ ) either before or after stress imaging, and the study parameters (cardiac biomarkers, cardiac chamber sizes, segmental defect scores) were compared between those with $(n=23)$ and without $(n=11)$ evidence of detectable NGAL. Analyses were conducted using SPSS version 16.0 (SPSS Inc., Chicago, Illinois) and $P<.05$ was required for statistical significance. Unless otherwise specified, values are expressed as mean \pm standard deviation.

\section{Results}

There were 16 and 18 subjects in the low and high inducible ischemia groups, respectively. The groups were similar for all baseline demographic, laboratory, and cardiovascular parameters, with the exception of prior history of coronary angioplasty, which was more prevalent in the high inducible ischemia group (Table 1). Plasma albumin and hemoglobin fell significantly two hours after stress testing $(P<.001)$.

Changes in cardiac biomarkers, oxidative stress, and various cardiovascular imaging parameters compared between the high and low inducible ischemia groups are presented in Table 2. Patients in the high ischemia group demonstrated a consistent rise in cardiac biomarkers with stress. For example, the rise in BNP following stress was $8.0 \mathrm{pg} / \mathrm{mL}$ greater in the high ischemia than the low ischemia group (delta-BNP $11.8 \mathrm{pg} / \mathrm{mL}$ versus $3.8 \mathrm{pg} / \mathrm{mL}, 95 \%$ confidence interval $1.2-$ $14.7 ; P=.02)$. A similar pattern was seen with NT-proBNP, which increased more in the high ischemia group compared to the low ischemia group (delta NT-proBNP $59.1 \mathrm{pg} / \mathrm{mL}$ versus $5.0 \mathrm{pg} / \mathrm{mL}, 95 \%$ confidence interval $4.9-103.3 ; P=$ .03). Likewise, the poststress cTnI rose to $0.016 \mu \mathrm{g} / \mathrm{L}$ in the high ischemia group and to $0.008 \mu \mathrm{g} / \mathrm{L}$ in the low ischemia group $(P=.05)$. A trend was seen with respect to change in end-diastolic volume, which increased in the high ischemia group by $6.5 \mathrm{~mL}$ versus $0.1 \mathrm{~mL}$ in the low ischemia group $(P=.07)$. There also was a weaker trend towards higher oxidative stress in the high ischemia group, with AOPP of $121.2 \mu \mathrm{mol} / \mathrm{L}$ in the high ischemia versus $77.0 \mu \mathrm{mol} / \mathrm{L}$ in the low ischemia group $(P=.15)$.

Plasma NGAL levels were below the limits of detection in many instances, both before and after stress, hence preand postcomparisons could not be made between groups based on their level of inducible ischemia. However, when the subjects were divided into two groups based on the presence or absence of detectable NGAL levels $(\geq 60 \mathrm{ng} / \mathrm{mL})$, before or after stress imaging, some clear differences emerged as presented in Table 3. Both plasma BNP and NT-proBNP rose with stress to a greater degree in the detectable NGAL group, and this was statistically significant in the former case, with BNP rising by $6.7 \mathrm{pg} / \mathrm{mL}$ more in the NGAL group (95\% confidence interval $1.0-14.4 \mathrm{pg} / \mathrm{mL} ; P=.03$ ). Levels of $\mathrm{cTnI}$ at baseline, prior to stress imaging, were higher in the detectable NGAL group, at 0.010 versus $0.006 \mu \mathrm{g} / \mathrm{L}$ $(P=.05)$. The subjects with detectable NGAL also had a significantly higher segmental defect score at rest, and higher end-diastolic and end-systolic volumes at rest and with stress. Ejection fraction was significantly lower both at rest and with stress in patients with detectable NGAL (all $P<.05$, Table 2 ).

\section{Discussion}

We designed this study to examine for evidence of cardiorenal signaling in patients with varying degrees of inducible cardiac ischemia and diminished systolic function. We clearly identified that those subjects with higher degrees of inducible ischemia had a consistent increase in a biomarker of cardiac injury (cTnI) and biomarkers of cardiac distress (BNP and NT-proBNP). Furthermore, the patients with lower LVEF had detectable plasma NGAL, that is, an increase in a biomarker of kidney injury.

Biomarkers are biological substances of human physiology, reflecting change in function or appearance of injury in certain organ or system of organs. The ideal biomarker is easily measured, specific for the organ under inspection, appears early after injury, shows the amount of injury, and correlates with prognosis [24]. In the discipline of cardiology, the cardiac troponins have been shown to possess these properties. In nephrology, early biomarkers indicating kidney injury have long been longed for and have only recently become available for clinical use [25].

In addition to cardiac troponins, B-type natriuretic peptides have gained success as diagnostic and prognostic biomarkers, especially among CHF patients [26]. BNPs are secreted by the cardiac ventricles in response to excessive stretching of myocytes, in heart failure and volume overload, and ischemic injury to myocardium [27]. Importantly, in earlier studies elevated levels of BNPs have been shown to be independent predictors of cardiovascular morbidity and mortality, both in patients with normal and impaired renal function, thus emphasizing the value of BNPs in assessment of cardiorenal syndrome $[1,10]$. In our study, the levels of both BNP and NT-proBNP were significantly higher in patients with more myocardial ischemic perfusion defects.

Neutrophil gelatinase-associated lipocalin (NGAL) has emerged as a novel biomarker of acute kidney injury (AKI) [15]. NGAL is a $25-\mathrm{kDa}$ protein widely spread within human body (kidney, prostate, uterus, salivary gland, epithelia of respiratory, and alimentary tracts) and shown to possess various biological properties, for instance kidney-protecting and nephron-inducing activity and bacteriostatic capability [15]. Although normally expressed at very low levels, it has been shown to rise in AKI, in human kidney cortical tubules, urine, and plasma and has therefore become a novel biomarker of acute renal damage [28]. Furthermore, the rise of NGAL takes place faster than a possible increase in plasma creatinine allowing detection of AKI earlier than with creatinine-based criteria and with good sensitivity and excellent specificity [29]. NGAL has been shown to increase in various settings of cardiac procedures and in critically ill patients with sepsis, renal ischemia and contrast mediainduced nephropathy [30]. The acute rise of NGAL in most reported studies has taken place during two to six hours after an event compromising renal function and causing renal damage [15].

Interestingly, NGAL has been shown to be elevated in patients with CHF, possibly demonstrating a link between cardiac dysfunction and renal injury $[16,17]$. This is in line with the results of our study, in which the patients with 
TABLE 1: Baseline demographic, laboratory, and cardiovascular parameters according to the amount of myocardial ischemia (number of inducible myocardial perfusion defects).

\begin{tabular}{|c|c|c|c|}
\hline Baseline variable & High inducible ischemia $(n=18)$ & Low inducible ischemia $(n=16)$ & $P$ value \\
\hline \multicolumn{4}{|l|}{ Demographic } \\
\hline Age (years) & $70.4 \pm 6.7$ & $67.6 \pm 7.5$ & .25 \\
\hline Sex $(\%$ male $)$ & 77.8 & 75.0 & .85 \\
\hline Weight (kg) & $80.5 \pm 12.1$ & $78.1 \pm 8.0$ & .66 \\
\hline \multicolumn{4}{|l|}{ Past history (\%) } \\
\hline Hypertension & 38.9 & 43.8 & .77 \\
\hline Current smoking & 0.0 & 18.8 & .09 \\
\hline Dyslipidemia & 66.7 & 56.3 & .53 \\
\hline Diabetes mellitus & 22.2 & 12.5 & .46 \\
\hline Myocardial infarction & 77.8 & 43.8 & .08 \\
\hline Coronary angioplasty & 88.9 & 50.0 & .02 \\
\hline Coronary bypass graft & 5.6 & 12.5 & .48 \\
\hline \multicolumn{4}{|l|}{ Medications (\%) } \\
\hline Nitrates & 16.7 & 18.8 & .87 \\
\hline Calcium antagonists & 33.3 & 12.5 & .23 \\
\hline Beta blockers & 27.8 & 31.3 & .82 \\
\hline \multicolumn{4}{|l|}{ Laboratory } \\
\hline Creatinine $(\mu \mathrm{mol} / \mathrm{L})$ & $86.0 \pm 27.2$ & $75.6 \pm 16.6$ & .19 \\
\hline Albumin (g/L) & $45.3 \pm 2.3$ & $44.6 \pm 2.4$ & .39 \\
\hline Hemoglobin (g/L) & $140.7 \pm 13.4$ & $147.0 \pm 13.5$ & .18 \\
\hline Glucose (mmol/L) & $6.4 \pm 1.0$ & $5.9 \pm 0.7$ & .15 \\
\hline $\mathrm{BNP}(\mathrm{pg} / \mathrm{mL})$ & $80.3 \pm 60.6$ & $52.9 \pm 43.9$ & .15 \\
\hline $\mathrm{cTnI}(\mu \mathrm{g} / \mathrm{L})$ & $0.010 \pm 0.009$ & $0.008 \pm 0.007$ & .45 \\
\hline NT-proBNP (pg/mL) & $580.4 \pm 712.9$ & $289.9 \pm 215.8$ & .13 \\
\hline $\mathrm{AOPP}(\mu \mathrm{mol} / \mathrm{L})$ & $121.2 \pm 116.3$ & $77.0 \pm 28.5$ & .15 \\
\hline NGAL $(\mathrm{ng} / \mathrm{mL})^{\dagger}$ & $67.5(60.0-115.5)$ & $67.0(60.0-90.8)$ & .60 \\
\hline \multicolumn{4}{|c|}{ Cardiovascular parameters (at rest) } \\
\hline End diastolic volume (mL) & $101.3 \pm 29.7$ & $105.2 \pm 27.9$ & .70 \\
\hline End systolic volume (mL) & $46.5 \pm 24.5$ & $43.8 \pm 19.1$ & .73 \\
\hline Ejection fraction $(\%)$ & $56.7 \pm 10.6$ & $61.1 \pm 12.1$ & .27 \\
\hline
\end{tabular}

${ }^{\dagger}$ Data presented as median (IQR).

BNP: B-type natriuretic peptide, cTnI: cardiac troponin I, NT-proBNP: N-terminal-pro-B-type natriuretic peptide, AOPP: advanced oxidation protein products, NGAL: neutrophil gelatinase-associated lipocalin.

lower LVEF both at rest and with stress had detectable plasma NGAL, thus suggesting similar association between chronic heart failure and continuous kidney damage. The level of NGAL has also been shown to correlate with severity and progression of chronic kidney disease [31], which further increases the prognostic importance of elevated NGAL. Whether or not the damaged heart can be said to be "signaling" the kidneys through mechanisms independent of hemodynamic factors is the subject of ongoing study. Future work may reveal if NGAL is a useful biomarker in the evaluation of patients presenting with cardiorenal syndromes.

While our experimental model demonstrated a measurable cardiorenal signal in the context of cardiac ischemia and diminished LVEF with higher levels of BNPs, a secondary aim was to identify evidence that the relationship between cardiac ischemia and the kidneys was injurious to the kidneys (type 1 or 2 cardiorenal syndrome). To this end, we measured plasma NGAL as a marker of kidney injury. Subjects with detectable levels of NGAL in the plasma demonstrated greater incremental changes in natriuretic peptides in response to stress, had more segmental perfusion defects, higher end-diastolic and end-systolic volumes and lower LVEF with stress, and had higher baseline levels of the cardiac injury biomarker cTnI. Serum creatinine levels did not differ significantly between the groups before or after stress. While these observations are associations and do not speak specifically to mechanism, one plausible conclusion is that myocardial ischemia was contributing to a mild, subclinical level of kidney injury. In addition, diminished LVEF could be an injurious factor to renal tubular cells causing continuous, prolonged kidney injury. An alternative 
TABLE 2: Changes in cardiac biomarkers, oxidative stress, and various cardiovascular imaging parameters compared between the high and low ischemia (number of inducible myocardial perfusion defects) groups.

\begin{tabular}{|c|c|c|c|}
\hline Study variable & High inducible ischemia $(n=18)$ & Low inducible ischemia $(n=16)$ & $P$ value \\
\hline \multicolumn{4}{|l|}{ Laboratory } \\
\hline Creatinine $(\mu \mathrm{mol} / \mathrm{L})$ —at rest & $86.0 \pm 27.2$ & $75.6 \pm 16.6$ & .19 \\
\hline Creatinine $(\mu \mathrm{mol} / \mathrm{L})$ — with stress & $88.2 \pm 28.6$ & $78.5 \pm 18.0$ & .25 \\
\hline$\Delta$ Creatinine $(\mu \mathrm{mol} / \mathrm{L})$ & $2.2 \pm 7.3$ & $2.9 \pm 8.0$ & .80 \\
\hline $\mathrm{BNP}(\mathrm{pg} / \mathrm{mL})$ - at rest & $80.3 \pm 60.6$ & $52.9 \pm 43.9$ & .15 \\
\hline $\mathrm{BNP}(\mathrm{pg} / \mathrm{mL})$ —with stress & $92.1 \pm 68.8$ & $56.8 \pm 40.3$ & .08 \\
\hline$\Delta \mathrm{BNP}(\mathrm{pg} / \mathrm{mL})$ & $11.8 \pm 11.8$ & $3.8 \pm 7.2$ & .02 \\
\hline$c \operatorname{TnI}(\mu \mathrm{g} / \mathrm{L})$-at rest & $0.010 \pm 0.009$ & $0.008 \pm 0.007$ & .45 \\
\hline$c \operatorname{TnI}(\mu \mathrm{g} / \mathrm{L})$ - with stress & $0.016 \pm 0.014$ & $0.008 \pm 0.007$ & .05 \\
\hline$\Delta \mathrm{cTnI}(\mu \mathrm{g} / \mathrm{L})$ & $0.007 \pm 0.013$ & $0.002 \pm 0.004$ & .16 \\
\hline NT-proBNP $(\mathrm{pg} / \mathrm{mL})$ —at rest & $580.4 \pm 712.9$ & $289.9 \pm 215.8$ & .13 \\
\hline NT-proBNP (pg/mL) — with stress & $639.5 \pm 754.1$ & $294.9 \pm 204.3$ & .08 \\
\hline$\Delta \mathrm{NT}$-proBNP $(\mathrm{pg} / \mathrm{mL})$ & $59.1 \pm 84.9$ & $5.0 \pm 48.8$ & .03 \\
\hline $\operatorname{AOPP}(\mu \mathrm{mol} / \mathrm{L})$-at rest & $121.2 \pm 116.3$ & $77.0 \pm 28.5$ & .15 \\
\hline $\mathrm{AOPP}(\mu \mathrm{mol} / \mathrm{L})$ — with stress & $95.0 \pm 102.2$ & $65.2 \pm 18.6$ & .26 \\
\hline$\Delta \mathrm{AOPP}(\mu \mathrm{mol} / \mathrm{L})$ & $-26.1 \pm 49.4$ & $-11.8 \pm 26.2$ & .29 \\
\hline \multicolumn{4}{|l|}{ Cardiovascular parameters } \\
\hline Segmental perfusion defects - at rest & $3.8 \pm 5.5$ & $1.8 \pm 3.5$ & .22 \\
\hline Segmental perfusion defects—with stress & $10.7 \pm 7.1$ & $2.6 \pm 4.0$ & $<.001$ \\
\hline$\Delta$ Segmental perfusion defects & $6.6 \pm 3.0$ & $0.8 \pm 0.8$ & $<.001$ \\
\hline End diastolic volume $(\mathrm{mL})$ - at rest & $101.3 \pm 29.7$ & $105.2 \pm 27.9$ & .70 \\
\hline End diastolic volume $(\mathrm{mL})$ — with stress & $107.8 \pm 36.7$ & $102.2 \pm 27.4$ & .63 \\
\hline$\Delta$ End diastolic volume $(\mathrm{mL})$ & $6.5 \pm 10.9$ & $0.1 \pm 8.8$ & .07 \\
\hline End systolic volume $(\mathrm{mL})$ —at rest & $46.5 \pm 24.5$ & $43.8 \pm 19.1$ & .73 \\
\hline End systolic volume $(\mathrm{mL})$ - with stress & $51.1 \pm 30.4$ & $42.3 \pm 19.7$ & .34 \\
\hline$\Delta$ End systolic volume $(\mathrm{mL})$ & $4.6 \pm 8.7$ & $0.4 \pm 8.6$ & .19 \\
\hline Ejection fraction (\%) —at rest & $56.7 \pm 10.6$ & $61.1 \pm 12.1$ & .27 \\
\hline Ejection fraction $(\%)$ - with stress & $55.6 \pm 11.6$ & $60.6 \pm 11.0$ & .21 \\
\hline$\Delta$ Ejection fraction $(\%)$ & $-1.1 \pm 3.9$ & $-0.5 \pm 6.1$ & .74 \\
\hline
\end{tabular}

$\Delta$ : delta (change from pre-stress to poststress test).

BNP: B-type natriuretic peptide, cTnI: cardiac troponin I, NT-proBNP: N-terminal-pro-B-type natriuretic peptide, AOPP: advanced oxidation protein products, NGAL: neutrophil gelatinase-associated lipocalin.

explanation is that subjects with evidence of kidney injury were predisposed to cardiac ischemia and/or decreased systolic function (renocardiac syndrome).

There are a few limitations in our study that merit discussion. Firstly, plasma NGAL was below the limits of detection in many instances, and hence lacked sensitivity to detect subtle degrees of AKI following myocardial stress and ischemia. Plasma and urinary NGAL have been shown to be sensitive and specific indicators and predictive biomarkers of acute kidney injury, in for instance, adult intensive care unit patients with sepsis, children undergoing cardiopulmonary bypass, adults with cardiac surgery, and in patients with contrast-induced nephropathy [32]. NGAL has performed especially well in relatively uncomplicated patient populations with AKI, with excellent receiveroperating characteristics $[14,32]$. However, plasma NGAL measurement may be influenced by several confounding factors, such as systemic infections and inflammatory and malignant processes [32], for which our study population was not examined. It is also possible that in our study not enough time had elapsed following stress for NGAL to rise, as most studies examining the kinetics of plasma NGAL seem to indicate that a significant increase takes places more than two hours following the index event [15]. Future studies should consider using a panel of plasma and urinary biomarkers for AKI and follow subjects for a longer period of time. Secondly, the possible influence of dipyridamole on biomarkers such as NGAL and markers of oxidative stress should be taken into consideration. As a substance with antioxidative and antiapoptotic properties [33,34], some of our results may have suffered interference by this agent. However, the low and high ischemia groups were well balanced in terms of their 
TABLE 3: Results between groups based on existence of detectable plasma neutrophil gelatinase-associated lipocalin (NGAL).

\begin{tabular}{|c|c|c|c|}
\hline Study variable & Detectable NGAL $(n=23)$ & No detectable NGAL $(n=11)$ & $P$ value \\
\hline \multicolumn{4}{|l|}{ Laboratory } \\
\hline Creatinine $(\mu \mathrm{mol} / \mathrm{L})$ —at rest & $84.0 \pm 25.2$ & $75.1 \pm 17.5$ & .24 \\
\hline Creatinine $(\mu \mathrm{mol} / \mathrm{L})$ — with stress & $87.4 \pm 26.0$ & $75.9 \pm 19.4$ & .16 \\
\hline$\Delta$ Creatinine $(\mu \mathrm{mol} / \mathrm{L})$ & $3.3 \pm 8.1$ & $0.8 \pm 6.1$ & .32 \\
\hline $\mathrm{BNP}(\mathrm{pg} / \mathrm{mL})$ - at rest & $73.8 \pm 57.6$ & $53.9 \pm 46.7$ & .29 \\
\hline $\mathrm{BNP}(\mathrm{pg} / \mathrm{mL})$ —with stress & $84.0 \pm 64.3$ & $57.5 \pm 44.0$ & .17 \\
\hline$\Delta \mathrm{BNP}(\mathrm{pg} / \mathrm{mL})$ & $10.2 \pm 11.9$ & $3.5 \pm 4.8$ & .03 \\
\hline $\mathrm{cTnI}(\mu \mathrm{g} / \mathrm{L})$ - at rest & $0.010 \pm 0.009$ & $0.006 \pm 0.003$ & .05 \\
\hline$c \operatorname{TnI}(\mu \mathrm{g} / \mathrm{L})$-with stress & $0.013 \pm 0.010$ & $0.012 \pm 0.016$ & .86 \\
\hline$\Delta c \operatorname{TnI}(\mu \mathrm{g} / \mathrm{L})$ & $0.003 \pm 0.007$ & $0.007 \pm 0.015$ & .39 \\
\hline NT-proBNP $(\mathrm{pg} / \mathrm{mL})$ —at rest & $511.0 \pm 642.0$ & $303.1 \pm 258.0$ & .19 \\
\hline NT-proBNP (pg/mL)—with stress & $556.3 \pm 685.6$ & $312.1 \pm 226.5$ & .13 \\
\hline$\Delta \mathrm{NT}-$ proBNP $(\mathrm{pg} / \mathrm{mL})$ & $45.4 \pm 83.2$ & $9.0 \pm 46.0$ & .11 \\
\hline $\mathrm{AOPP}(\mu \mathrm{mol} / \mathrm{L})$ —at rest & $105.7 \pm 102.0$ & $89.3 \pm 52.8$ & .54 \\
\hline AOPP $(\mu \mathrm{mol} / \mathrm{L})$ — with stress & $85.8 \pm 91.1$ & $71.1 \pm 24.7$ & .48 \\
\hline$\Delta \mathrm{AOPP}(\mu \mathrm{mol} / \mathrm{L})$ & $-19.9 \pm 41.9$ & $-18.2 \pm 38.6$ & .91 \\
\hline \multicolumn{4}{|l|}{ Cardiovascular parameters } \\
\hline Segmental perfusion defects—at rest & $3.8 \pm 5.3$ & $0.9 \pm 2.1$ & .03 \\
\hline Segmental perfusion defects—with stress & $8.2 \pm 8.0$ & $4.2 \pm 3.7$ & .06 \\
\hline$\Delta$ Segmental perfusion defects & $4.1 \pm 3.8$ & $3.3 \pm 3.3$ & .51 \\
\hline End diastolic volume $(\mathrm{mL})$ - at rest & $108.4 \pm 25.9$ & $90.8 \pm 31.7$ & .14 \\
\hline End diastolic volume $(\mathrm{mL})$ — with stress & $112.8 \pm 32.5$ & $90.2 \pm 27.9$ & .05 \\
\hline$\Delta$ End diastolic volume $(\mathrm{mL})$ & $4.8 \pm 11.2$ & $0.8 \pm 8.0$ & .25 \\
\hline End systolic volume $(\mathrm{mL})$ —at rest & $49.9 \pm 21.5$ & $34.7 \pm 20.1$ & .07 \\
\hline End systolic volume (mL)—with stress & $53.5 \pm 27.5$ & $34.4 \pm 18.0$ & .02 \\
\hline$\Delta$ End systolic volume $(\mathrm{mL})$ & $3.6 \pm 9.4$ & $1.1 \pm 7.4$ & .44 \\
\hline Ejection fraction (\%)—at rest & $55.8 \pm 10.6$ & $65.0 \pm 10.7$ & .03 \\
\hline Ejection fraction (\%)—with stress & $55.1 \pm 11.0$ & $63.8 \pm 10.3$ & .04 \\
\hline$\Delta$ Ejection fraction $(\%)$ & $-0.7 \pm 5.1$ & $-1.2 \pm 4.9$ & .78 \\
\hline
\end{tabular}

$\Delta$ : delta (change from prestress to poststress test).

BNP: B-type natriuretic peptide, cTnI: cardiac troponin I, NT-proBNP: N-terminal-pro-B-type natriuretic peptide, AOPP: advanced oxidation protein products, NGAL: neutrophil gelatinase-associated lipocalin.

exposure to dipyridamole, hence exposure should not have led to spurious findings in one group over another. Likewise, the administration of aminophylline (a nonselective adenosine receptor antagonist) as part of the stress imaging protocol may have indirectly interfered with our results. Adenosine can affect renal function through its effects on renal blood flow and tubuloglomerular feedback $[35,36]$, however the likelihood of exposure to aminophylline was similar in both high and low ischemia groups. Thirdly, the observations made in this study do not clearly provide a mechanism by which the ischemic heart is signaling the kidneys (or vice versa). While the endocrine effects of natriuretic peptides as signaling agents are not in dispute, one would not expect these to invoke kidney injury per se. Having identified that there does seem to be a relationship between the ischemic heart, and the heart with diminished systolic function, with a biomarker of kidney injury, further studies examining the role of cytokines, neurohormones, mediators of apoptosis, and other injurious pathways are planned. Additional limitations of our study are the rather low number of patients, and the number of measurements of plasma NGAL during the timecourse of the stress testing. Using multiple measurements, increasing the sample size and extending duration of posttest followup might have presented with a more distinct and positive rise in NGAL levels. Furthermore, the changes in the levels of cardiac biomarkers, while rather low in a clinical sense, were nonetheless statistically significant. We believe this represents a weak yet discernible signal as evidence for heart-kidney interactions.

To conclude, to our knowledge our study is novel in demonstrating the association between the amount of inducible myocardial ischemia with cardiorenal signaling and biomarker of kidney injury. Furthermore, we present the novel finding of the association between lower LVEF and detectable NGAL in patients undergoing cardiac stress 
testing. Our results display intriguing, yet at present only suggestive insights into the signaling between the heart and the kidneys. Further studies to elucidate mechanisms at play in the complex bidirectional cardiorenal syndrome are ongoing.

\section{Abbreviations}

$\begin{array}{ll}\text { AKI: } & \text { Acute kidney injury } \\ \text { AOPP: } & \text { Advanced oxidation protein products } \\ \text { BNP: } & \text { Brain or B-type natriuretic peptide } \\ \text { CAD: } & \text { Coronary artery disease } \\ \text { CHF: } & \text { Congestive heart failure } \\ \text { cTnI: } & \text { Cardiac troponin I } \\ \text { LVEF: } & \text { Left ventricular ejection fraction } \\ \text { NGAL: } & \begin{array}{l}\text { Neutrophil gelatinase-associated } \\ \text { lipocalin }\end{array} \\ \text { NT-proBNP: } & \begin{array}{l}\text { N-terminal-pro-B-type natriuretic } \\ \text { peptide. }\end{array}\end{array}$

\section{Acknowledgment}

The authors are grateful for the assistance of staff in St. Bortolo Hospital Nuclear Medicine Department in carrying out this study. Biosite Inc. (San Diego, CA, USA) provided the NGAL kits, as they were not commercially available at the time of the study.

\section{References}

[1] C. Ronco, M. Haapio, A. A. House, N. Anavekar, and R. Bellomo, "Cardiorenal syndrome," Journal of the American College of Cardiology, vol. 52, no. 19, pp. 1527-1539, 2008.

[2] C. Ronco, P. McCullough, S. D. Anker et al., "Cardio-renal syndromes: report from the consensus conference of the acute dialysis quality initiative," European Heart Journal, vol. 31, no. 6, pp. 703-711, 2010.

[3] L. B. Daniels and A. S. Maisel, "Natriuretic peptides," Journal of the American College of Cardiology, vol. 50, no. 25, pp. 23572368, 2007.

[4] M. A. Burke and W. G. Cotts, "Interpretation of B-type natriuretic peptide in cardiac disease and other comorbid conditions," Heart Failure Reviews, vol. 12, no. 1, pp. 23-36, 2007.

[5] P. A. McCullough, R. M. Nowak, J. McCord et al., "B-type natriuretic peptide and clinical judgment in emergency diagnosis of heart failure: analysis from Breathing Not Properly (BNP) Multinational Study," Circulation, vol. 106, no. 4, pp. 416-422, 2002.

[6] T. Omland, "Advances in congestive heart failure management in the intensive care unit: B-type natriuretic peptides in evaluation of acute heart failure," Critical Care Medicine, vol. 36, no. 1, supplement, pp. S17-S27, 2008.

[7] D. Farmakis, G. Filippatos, M. Tubaro et al., "Natriuretic peptides in acute coronary syndromes: prognostic value and clinical implications," Congestive Heart Failure, vol. 14, no. 4, supplement 1, pp. 25-29, 2008.

[8] T. Omland, "B-type natriuretic peptides: prognostic markers in stable coronary artery disease," Expert Review of Molecular Diagnostics, vol. 8, no. 2, pp. 217-225, 2008.
[9] G. W. Moe, "B-type natriuretic peptide in heart failure," Current Opinion in Cardiology, vol. 21, no. 3, pp. 208-214, 2006.

[10] C. Bruch, C. Fischer, J. Sindermann, J. Stypmann, G. Breithardt, and R. Gradaus, "Comparison of the prognostic usefulness of N-terminal pro-brain natriuretic peptide in patients with heart failure with versus without chronic kidney disease," American Journal of Cardiology, vol. 102, no. 4, pp. 469-474, 2008.

[11] W. J. Austin, V. Bhalla, I. Hernandez-Arce et al., "Correlation and prognostic utility of B-type natriuretic peptide and its amino-terminal fragment in patients with chronic kidney disease," American Journal of Clinical Pathology, vol. 126, no. 4, pp. 506-512, 2006.

[12] S. J. Carr, S. Bavanandan, B. Fentum, and L. Ng, "Prognostic potential of brain natriuretic peptide (BNP) in predialysis chronic kidney disease patients," Clinical Science, vol. 109, no. 1, pp. 75-82, 2005.

[13] M. Svensson, A. Gorst-Rasmussen, E. B. Schmidt, K. A. Jorgensen, and J. H. Christensen, "NT-pro-BNP is an independent predictor of mortality in patients with end-stage renal disease," Clinical Nephrology, vol. 71, no. 4, pp. 380-386, 2009.

[14] J. Mishra, C. Dent, R. Tarabishi et al., "Neutrophil gelatinaseassociated lipocalin (NGAL) as a biomarker for acute renal injury after cardiac surgery," Lancet, vol. 365, no. 9466, pp. 1231-1238, 2005.

[15] P. Devarajan, "Neutrophil gelatinase-associated lipocalin-an emerging troponin for kidney injury," Nephrology Dialysis Transplantation, vol. 23, no. 12, pp. 3737-3743, 2008.

[16] K. Damman, D. J. van Veldhuisen, G. Navis, A. A. Voors, and H. L. Hillege, "Urinary neutrophil gelatinase associated lipocalin (NGAL), a marker of tubular damage, is increased in patients with chronic heart failure," European Journal of Heart Failure, vol. 10, no. 10, pp. 997-1000, 2008.

[17] B. Poniatowski, J. Malyszko, H. Bachorzewska-Gajewska, J. S. Malyszko, and S. Dobrzycki, "Serum neutrophil gelatinaseassociated lipocalin as a marker of renal function in patients with chronic heart failure and coronary artery disease," Kidney and Blood Pressure Research, vol. 32, no. 2, pp. 77-80, 2009.

[18] R. S. Foote, J. D. Pearlman, A. H. Siegel, and K.-T. J. Yeo, "Detection of exercise-induced ischemia by changes in Btype natriuretic peptides," Journal of the American College of Cardiology, vol. 44, no. 10, pp. 1980-1987, 2004.

[19] K. Kurz, R. Voelker, D. Zdunek et al., "Effect of stress-induced reversible ischemia on serum concentrations of ischemiamodified albumin, natriuretic peptides and placental growth factor," Clinical Research in Cardiology, vol. 96, no. 3, pp. 152$159,2007$.

[20] B. Hesse, K. Tägil, A. Cuocolo et al., "EANM/ESC procedural guidelines for myocardial perfusion imaging in nuclear cardiology," European Journal of Nuclear Medicine and Molecular Imaging, vol. 32, no. 7, pp. 855-897, 2005.

[21] R. C. Hendel, F. J. Wackers, D. S. Berman et al., "American Society of Nuclear Cardiology consensus statement: reporting of radionuclide myocardial perfusion imaging studies," Journal of Nuclear Cardiology, vol. 13, no. 6, pp. e152-e156, 2006.

[22] M. D. Cerqueira, N. J. Weissman, V. Dilsizian et al., "Standardized myocardial segmentation and nomenclature for tomographic imaging of the heart: a statement for healthcare professionals from the Cardiac Imaging Committee of the Council on Clinical Cardiology of the American Heart Association," Journal of Nuclear Cardiology, vol. 9, no. 2, pp. 240-245, 2002. 
[23] S. Kargotich, C. Goodman, D. Keast et al., "Influence of exercise-induced plasma volume changes on the interpretation of biochemical data following high-intensity exercise," Clinical Journal of Sport Medicine, vol. 7, no. 3, pp. 185-191, 1997.

[24] C. Ronco, A. A. House, and M. Haapio, "Cardiorenal syndrome: refining the definition of a complex symbiosis gone wrong," Intensive Care Medicine, vol. 34, no. 5, pp. 957-962, 2008.

[25] S. G. Coca, R. Yalavarthy, J. Concato, and C. R. Parikh, "Biomarkers for the diagnosis and risk stratification of acute kidney injury: a systematic review," Kidney International, vol. 73, no. 9, pp. 1008-1016, 2008.

[26] A. Maisel, C. Mueller, K. Adams Jr. et al., "State of the art: using natriuretic peptide levels in clinical practice," European Journal of Heart Failure, vol. 10, no. 9, pp. 824-839, 2008.

[27] G. E. Woodard and J. A. Rosado, "Recent advances in natriuretic peptide research: molecular Medicine," Journal of Cellular and Molecular Medicine, vol. 11, no. 6, pp. 1263-1271, 2007.

[28] V. S. Vaidya, M. A. Ferguson, and J. V. Bonventre, "Biomarkers of acute kidney injury," Annual Review of Pharmacology and Toxicology, vol. 48, pp. 463-493, 2008.

[29] J. M. Thurman and C. R. Parikh, "Peeking into the black box: new biomarkers for acute kidney injury," Kidney International, vol. 73, no. 4, pp. 379-381, 2008.

[30] K. Mori and K. Nakao, "Neutrophil gelatinase-associated lipocalin as the real-time indicator of active kidney damage," Kidney International, vol. 71, no. 10, pp. 967-970, 2007.

[31] D. Bolignano, A. Lacquaniti, G. Coppolino et al., "Neutrophil gelatinase-associated lipocalin (NGAL) and progression of chronic kidney disease," Clinical Journal of the American Society of Nephrology, vol. 4, no. 2, pp. 337-344, 2009.

[32] P. Devarajan, "Emerging urinary biomarkers in the diagnosis of acute kidney injury," Expert Opinion on Medical Diagnostics, vol. 2, no. 4, pp. 387-398, 2008.

[33] K. T. Kim, E.-J. Yeo, H. Choi, and S. C. Park, "The effect of pyrimidine nucleosides on adenosine-induced apoptosis in HL-60 cells," Journal of Cancer Research and Clinical Oncology, vol. 124, no. 9, pp. 471-477, 1998.

[34] S. Chakrabarti and J. E. Freedman, "Dipyridamole, cerebrovascular disease, and the vasculature," Vascular Pharmacology, vol. 48, no. 4-6, pp. 143-149, 2008.

[35] P. S. Modlinger and W. J. Welch, "Adenosine A1 receptor antagonists and the kidney," Current Opinion in Nephrology and Hypertension, vol. 12, no. 5, pp. 497-502, 2003.

[36] M. M. Dohadwala and M. M. Givertz, "Role of adenosine antagonism in the cardiorenal syndrome," Cardiovascular Therapeutics, vol. 26, no. 4, pp. 276-286, 2008. 


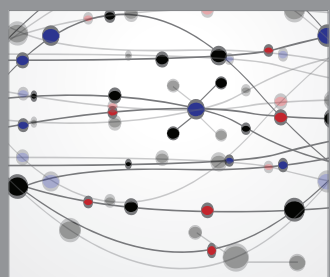

The Scientific World Journal
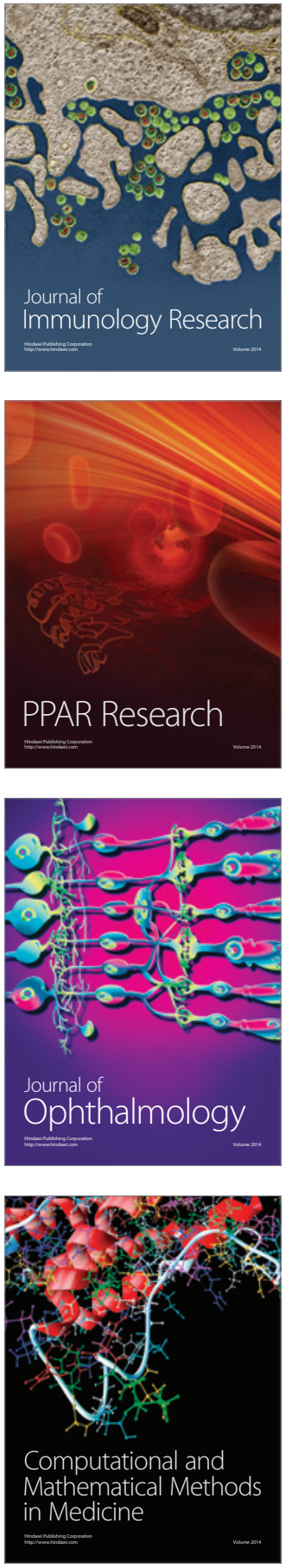

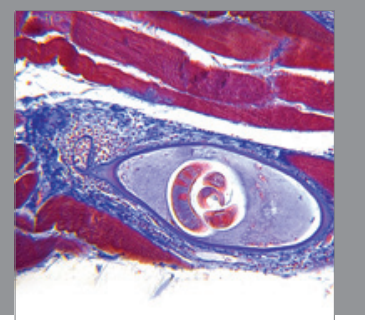

Gastroenterology

Research and Practice
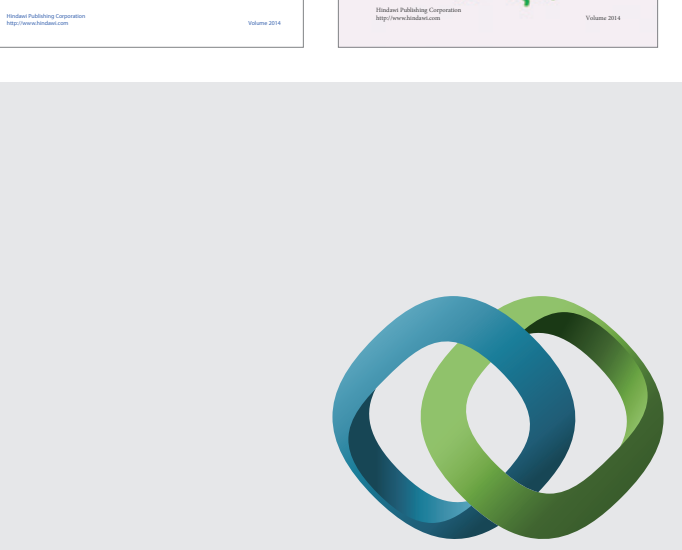

\section{Hindawi}

Submit your manuscripts at

http://www.hindawi.com
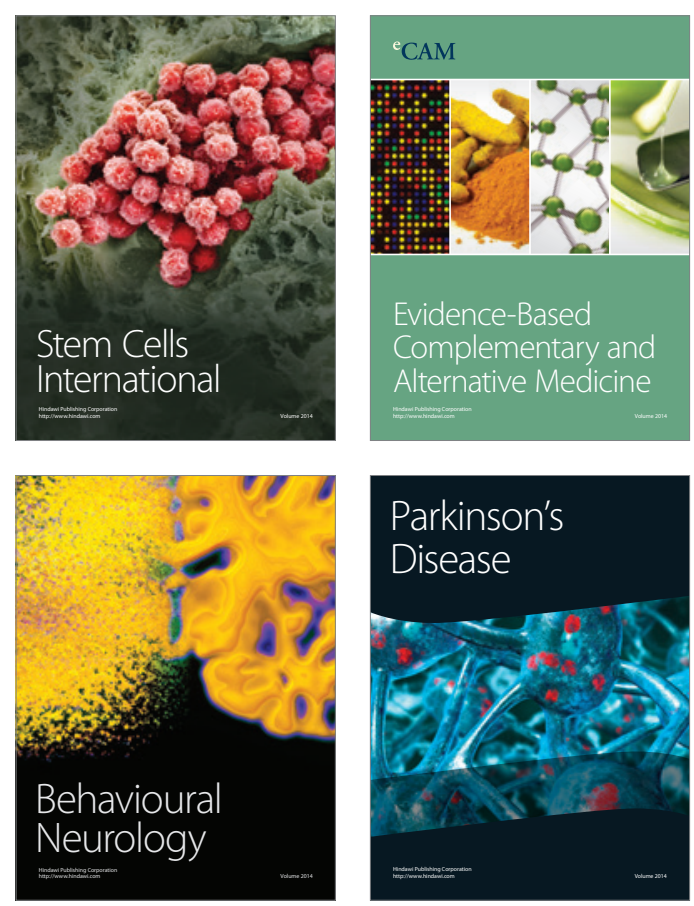

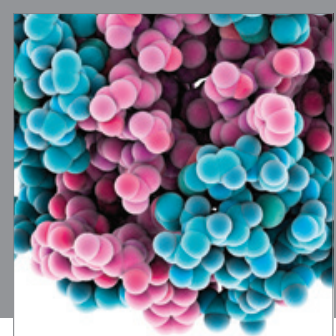

Journal of
Diabetes Research

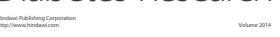

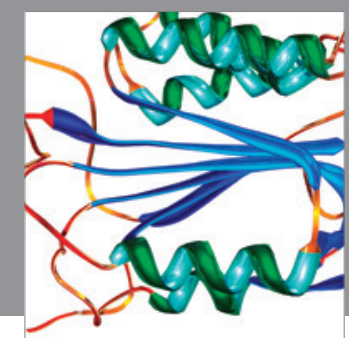

Disease Markers
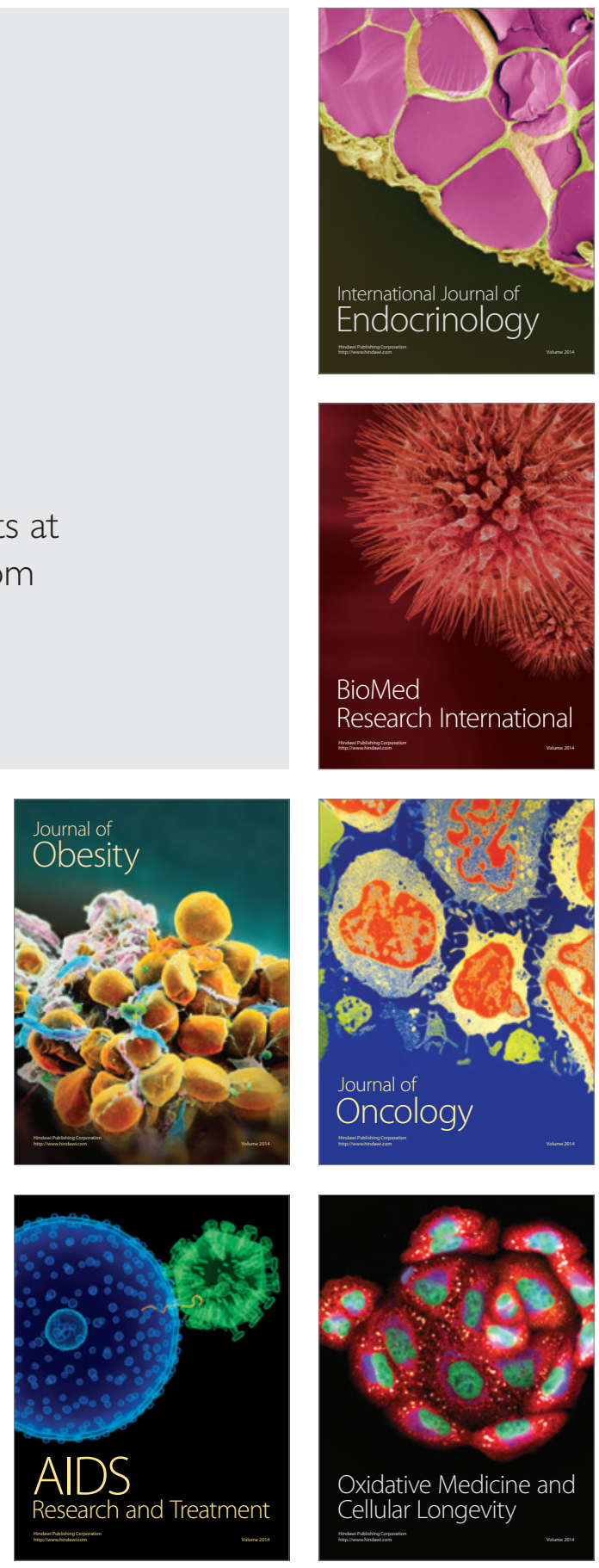